\title{
Recent Developments in SARS-CoV-2 Neutralizing Antibody Detection Methods
}

\author{
Jo-Lewis BANGA NDZOUBOUKOU, Yan-di ZHANG, Xiong-lin FAN \\ Department of Pathogen Biology, School of Basic Medicine, Tongji Medical College, Huazhong University of Science and \\ Technology, Wuhan 430030, China
}

(C) Huazhong University of Science and Technology 2021

\begin{abstract}
[Abstract] The ongoing Coronavirus disease 19 pandemic has likely changed the world in ways not seen in the past. Neutralizing antibody (NAb) assays play an important role in the management of the severe acute respiratory syndrome coronavirus-2 (SARS-CoV-2) outbreak. Using these tools, we can assess the presence and duration of antibody-mediated protection in naturally infected individuals, screen convalescent plasma preparations for donation, test the efficacy of immunotherapy, and analyze NAb titers and persistence after vaccination to predict vaccineinduced protective effects. This review briefly summarizes the various methods used for the detection of SARS-CoV-2 NAbs and compares their advantages and disadvantages to facilitate their development and clinical application.

Key words: Coronavirus disease 19; severe acute respiratory syndrome coronavirus-2; neutralizing antibodies; viral neutralization test; plaque reduction neutralization test; pseudovirus-based neutralization assays; enzyme-linked immunosorbent assay; lateral flow immunoassays
\end{abstract}

The ongoing Coronavirus disease 19 (COVID-19) pandemic may well have changed the world in ways not seen in the past. The World Health Organization (WHO) recently reported that more than 227 million people have been confirmed positive for severe acute respiratory syndrome coronavirus-2 (SARSCoV-2) infection, and at least 4.6 million deaths have been recorded ${ }^{[1]}$. SARS-CoV-2 represents the seventh human coronavirus $(\mathrm{HCoV})$ responsible for COVID-19 in humans. The other six types of HCoVs have been previously identified. These include $\mathrm{HCoV}-$ NL63 and HCoV-229E, which belong to the Alphacoronavirus $(\alpha-\mathrm{CoV})$ genus, $\mathrm{HCoV}-\mathrm{OC} 43, \mathrm{HCoV}-$ HKU1, SARS-CoV, middle east respiratory syndrome coronavirus (MERS-CoV), and SARS-CoV-2, which belong to the Beta-coronavirus $(\beta-\mathrm{CoV})$ genus $^{[2]}$. Among these, SARS-CoV-2, SARS-CoV, and MERS$\mathrm{CoV}$ are considered to be highly pathogenic ${ }^{[3]}$. Indeed, although COVID-19 has a lower mortality rate than SARS and MERS, the number of infections and deaths

Jo-Lewis BANGA NDZOUBOUKOU, E-mail: bangas19@ yahoo.fr

${ }^{\#}$ Corresponding author, E-mail: xlfan@hust.edu.cn

${ }^{*}$ This project was supported by grants from the Applied Basic Research Key Project of Wuhan Municipal Bureau of Science and Technology (2020020601012218) and the Fundamental Research Funds for the Central Universities (HUST COVID-19 Rapid Response Call No. 2020kfyXGYJ040). from COVID-19 is much higher than that recorded for SARS and MERS ${ }^{[4,5]}$. SARS-CoV-2 is transmitted from person-to-person through respiratory droplets and aerosols. However, some studies have shown that SARS-CoV-2 can be transmitted through contaminated objects or surfaces ${ }^{[6,7]}$. Approximately $81 \%$ of patients with COVID-19 have mild symptoms, while about $14 \%$ have severe symptoms such as dyspnea, high respiratory rate, and low oxygen saturation in the body's blood. Another group of patients (approximately $5 \%$ ), especially those over 60 years of age or with comorbidities, progress to critical conditions. About $3.4 \%$ of patients die from respiratory failure or multiorgan failure ${ }^{[8,9]}$. Thus, there is an urgent need to develop effective medical interventions to control the COVID-19 pandemic.

\section{SARS-COV-2 VIRAL STRUCTURE}

SARS-CoV-2 is an enveloped, positive-stranded RNA virus ${ }^{[10]}$. The SARS-CoV-2 genome size is approximately $29.8 \mathrm{kbp}$, making SARS-CoV-2 one of the largest RNA viruses ${ }^{[1-16]}$ (fig. 1A). Chan et al showed that the genomic sequence similarity of SARS-CoV-2 to SARS-CoV and bat SARS-like-CoV ZXC21 was $82 \%$ and $89 \%$, respectively ${ }^{[17]}$. The first $2 / 3$ of the genome contains a set of unstructured genes, which code specifically for enzymes associated with virus replication. The last $1 / 3$ encodes for a set of 
four structural proteins: the nucleocapsid $(\mathrm{N})$ protein containing the viral RNA and three structural proteins of the envelope, including the spike (S) protein, the envelope $(\mathrm{E})$ protein, and membrane $(\mathrm{M})$ protein $^{[18]}$ (fig. 1B). The $\mathrm{S}$ protein is mainly divided into two essential subunits: $\mathrm{S} 1$ and $\mathrm{S} 2$. The $\mathrm{S} 1$ subunit contains two main functional domains, the N-terminal domain (NTD) and the receptor-binding domain (RBD) ${ }^{[19]}$. The S2 subunit contains two repeat sequences heptapeptide 1 (HR1) and HR2 (fig. 1C). These domains typically play an essential role in viral adsorption and invasion of host cells. While the NTD and RBD structural domains facilitate the binding of $\mathrm{S}$ proteins to the receptor, the two structural domains of the S2 subunit are responsible for membrane fusion ${ }^{[19-21]}$. In addition, in the $\beta-\mathrm{CoV}$ genus, the $\mathrm{S} 2$ subunit is relatively more conserved, while the S1 subunit consisting of the RBD is much less conserved and often immunodominant ${ }^{[22]}$.

\section{SARS-COV-2 NEUTRALIZING ANTIBODY}

During a viral infection, the host triggers a humoral immune response characterized by the production of antibodies (Abs), also known as immunoglobulin (Ig).

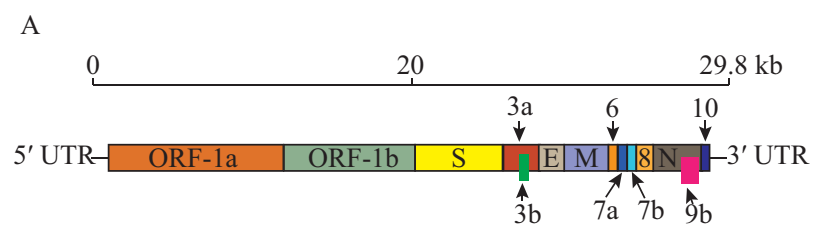

B

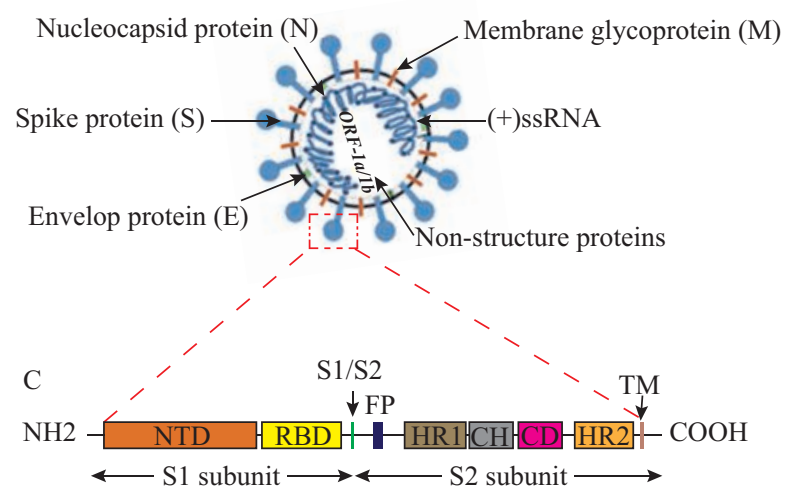

Fig. 1 SARS-CoV-2 viral structure model

A: genome structure. B: viral particle of SARS-CoV-2. The SARS-CoV-2 genome comprises of 5' and 3' untranslated region (UTR) and open reading frame (ORF) $1 \mathrm{a} / \mathrm{b}$. The structural genes present at the $3^{\prime}$ terminus encodes for the structural proteins including spike (S), membrane (M), nucleocapsid (N), and envelope (E). The accessory genes interspaced between the structural genes for accessory proteins. C: SARS-CoV-2 spike (S) glycoprotein. The S1 subunit contains a N-terminal domain (NTD) and receptor-binding domain (RBD). The $\mathrm{S} 2$ subunit contains the fusion peptide (FP), heptad repeat 1 (HR1), central helix $(\mathrm{CH})$, connector domain $(\mathrm{CD})$, heptad repeat 2 (HR2), and transmembrane domain (TM). Arrow S1/S2 represent the cleavage site.
The production of Abs is a significant function of the immune system, and these Abs are secreted from B cells (or B lymphocytes). It is known that initial exposure to an antigen $(\mathrm{Ag})$ (exposure can either result from infection or by vaccination), leads to the activation of naive $B$ cells. These B cells differentiate into the following two subgroups: antibody-producing plasma cells, which can produce Abs that travel through the blood, and memory B cells, which remember the antigen for future encounters. These Abs act like an army against viral infection. However, only a small subset of the Abs produced during viral infection, called neutralizing antibodies (NAbs), have the ability to prevent the virus from infecting host cells (fig. 2). Correspondingly, Abs can be classified into two groups, NAbs and nonneutralizing binding antibodies. Some studies show that the level of Abs in the blood varies considerably between individuals and over time after infection ${ }^{[23-27]}$. For example, Tang et al demonstrated that Abs were still detectable 6 years after SARS-CoV-1 infection ${ }^{[28]}$. Indeed, the duration and effectiveness of Abs are two essential parameters in the fight against the SARS$\mathrm{CoV}-2$ pandemic and the development of vaccines. To this end, several serological studies have assessed the dynamics and duration of $\mathrm{Ab}$ production and immunity after SARS-CoV-2 infections. The results from these studies are not uniform. Some studies suggest that there is a rapid decrease in Abs (often below the detection limit), while other studies suggest that $\mathrm{Ab}$ persistence occurs $^{[25,29-31]}$. Some studies showed that 20 days after the onset of symptoms, the levels of specific IgM and IgA against SARS-CoV-2 gradually decreased 3 to 5 months after infection ${ }^{[23,32,33]}$. Similar declines have also been reported in the context of MERS infection ${ }^{[34]}$ or other seasonal human coronaviruses, where the protective immunity appears to be short-lived ${ }^{[35-37]}$. IgM contributes to the early neutralization response ${ }^{[38]}$. Although IgM is the first line of humoral response, a peculiarity of SARS-CoV-2 infection is that all three isotypes (IgM, IgA, and $\operatorname{IgG}$ ) can be detected within a narrow time frame upon seroconversion ${ }^{[39-42]}$. It should be noted that all protein components of $\mathrm{HCoVs}$ are capable of inducing Abs. Before SARS-CoV-2 appeared, other HCoVs have been shown to elicit NAbs ${ }^{[43]}$. These NAbs probably offer at least some degree of protection against re-infection. Several studies have shown that the $\mathrm{HCoVs}$ protein $\mathrm{S}$ is the main target protein that stimulates the immune system to produce NAbs after infection ${ }^{[44-47]}$. For this purpose, various neutralizing monoclonal Abs against $\mathrm{HCoVs}$ protein $\mathrm{S}$ have been isolated and shown to be effective in animal models ${ }^{[48-51]}$.

Since the emergence of SARS-CoV-2, the RBD domain and the $\mathrm{S}$ protein are the main targets of humoral immune responses and represent the antigenic domain frequently used in most serological assays 
Antibodies (immunoglobulins) classification:

1) Immunoglobulin $M$ ( $(\operatorname{ggM})$;

2) Immunoglobulin $G(\operatorname{IgG})$;

3) Immunoglobulin A (IgA);

4) Immunoglobulin E (IgE);

5) Immunoglobulin $\mathrm{D}(\mathrm{IgD})$.

IgM is the first immunoglobulin produced by B-cells against new infection.

$\mathrm{IgG}$ is the most abundant antibody class. There are 4 subclasses: IgG1, IgG2, IgG3 and IgG4.

$\operatorname{IgA}$ is the most prevalent antibody in secretions, such as secretion and mucous. There are 2 subclasses: $\operatorname{IgA} 1$ and $\operatorname{IgA} 2$.

IgE is associated with allergy.

$\mathrm{IgD}$ is involved in B cells and antibody production.

SARS-CoV-2 (Pathogens)

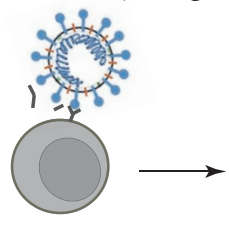

Naive B cells

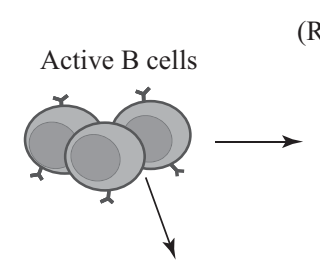

Memory B cells

(Remembering the $\mathrm{Ag}$ for future encounters)

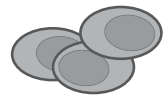

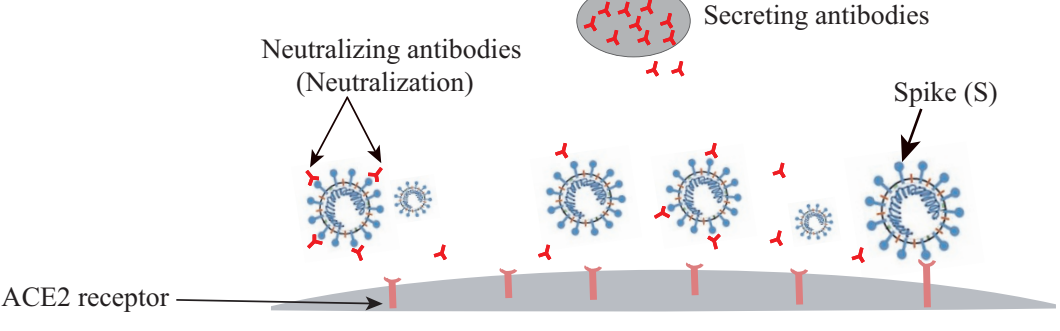

Host cells membrane

Fig. 2 Antibodies production process and neutralization

Antibodies are produced by B cells and their progeny. Naïve B cells in peripheral lymphoid tissues recognize antigen but do not secrete antibodies, and activation of these cells stimulates their differentiation into antibody-secreting plasma cells and memory cells. Neutralization of enveloped viruses blocks viral attachment and entry into target cells.

for COVID-19 ${ }^{[18,52,53]}$. Likewise, significant efforts have been made to develop vaccines. The main target of most of these vaccines is the RBD domain of the SARS-CoV-2 S protein to induce NAbs and prevent the RBD domain from interacting with the receptor angiotensin 2 converting enzyme (ACE2) on the host cell $^{[54]}$. Several vaccines, such as Moderna ${ }^{[55]}$ and Pfizer/BioNTech ${ }^{[56]}$, have been shown to be nearly $95 \%$ effective in their phase 3 clinical trials. Thus, SARS$\mathrm{CoV}-2$ NAbs can be produced during natural viral infection or vaccination. The $\mathrm{S}$ protein also appears to be the main target antigen in the development of the SARS-CoV-2 vaccines and the design of therapeutic products. Li et al first described the linear epitopes of the $\mathrm{S}$ protein, which were concentrated in two regions of the $\mathrm{S}$ protein, the $\mathrm{C}$-terminal domain, and a region close to the fusion peptide ${ }^{[57]}$. Surprisingly, the RBD region only had three moderate immunogenic epitope $^{[57]}$. Several studies involving a population positive for COVID-19 showed that nearly $90 \%$ of the NAbs specifically targeted the RBD domain of the S1 subunit of the $\mathrm{S}$ protein ${ }^{[58]}$. Therefore, among the four structural proteins of the SARS-CoV-2 virus, more NAbs are produced against the $\mathrm{S}$ protein. Furthermore, NAbs are essential in viral clearance and are often considered key immune products that can be used for protection or the development of therapeutic approaches against viral diseases. The presence of NAbs is a good indicator of protective immunity for most viral infections. Similarly, detecting NAbs during SARS$\mathrm{CoV}-2$ infection is evidence of protective immunity against SARS-CoV-2 ${ }^{[59]}$. In fact, SARS-CoV-2 NAbs play an important role in the host's defense against COVID-19 because the NAbs protect individuals from SARS-CoV-2 infection ${ }^{[60-62]}$. NAbs bind and abrogate the entry of SARS-CoV-2 S protein into host cells by recognizing the relevant ACE2 receptors present in host cells ${ }^{[62]}$. Moreover, Lei et al reported that NAb responses during the hospitalization of COVID-19 patients underscored clinical progression and outcomes, which also indicates the important role of NAb in the disease $^{[63]}$. Cao et al showed that monoclonal NAbs had a great therapeutic and prophylactic effect in human ACE2 transgenic mice infected with SARS$\mathrm{CoV}-2^{[64]}$. Therefore, the quantification of SARS$\mathrm{CoV}-2$-specific NAbs seems to be essential in order to control the COVID-19 pandemic at both medical and socioeconomic levels. In addition, detection of NAbs against SARS-CoV-2 will help to understand the status of protective immune responses in COVID-19 patients and in asymptomatic cases ${ }^{[65]}$. The assays that can measure NAbs produced during infection with SARS$\mathrm{CoV}-2$ are essential to understanding the pathogenesis of SARS-CoV-2 and to better study the response of 
the human immune system to SARS-CoV-2. Besides, a better understanding of the efficacy of NAbs and how they bind to target epitopes could be an essential pathway in the development of effective vaccines and thus improve immunotherapeutic agents. Therefore, in evaluating the efficacy of a vaccine, it is essential to determine the presence of NAbs and to measure the required level of these NAbs to prevent future exposure to the virus. With the introduction of a vaccine against COVID-19, various Ab tests will be able to learn all of this critical information. In fact, exposure to COVID-19 will be tested against the level of immunity and the immunity provided by the vaccine. In the fight against the COVID-19 pandemic, measuring SARS-CoV-2 NAbs is crucial for establishing a good diagnosis, for studying serological epidemiology, improving clinical management of SARS-CoV-2 infection ${ }^{[6]]}$, or aiding in the identification of potential Abs donors for convalescent plasma therapy ${ }^{[67]}$. In the present review, we briefly summarize the various methods currently used for the detection of SARS-CoV-2 NAbs.

\section{MAIN DETECTION METHODS OF SARS-CoV-2 NAbs}

Numerous assays have already been established to measure the neutralization capacity of $\mathrm{Abs}^{[68-71]}$. The viral neutralization test (VNT) is the current gold standard for the detection of NAbs. The VNT can be the plaque reduction neutralization test (PRNT), the pseudotyped viral neutralization test, or the microneutralization test. In fact, for NAbs detection, these tests are frequently used as serological methods. However, these tests often face several limitations. Indeed, their need to use live viruses and biosafety level 3 (BSL-3) containment facilities are not suitable for routine testing ${ }^{[72]}$, so commercial SARS-CoV-2 immunoassays need to be evaluated to determine if they can provide reliable information about serum neutralizing activity. Some of these immunoassays use indirect serological methods, such as the enzyme-linked immunosorbent assay (ELISA), immunofluorescence assay, and immunochromatography. These immunoassays use the principle of the $\mathrm{Ag}-\mathrm{Ab}$ reaction and have been established as sero-diagnostic methods for SARS-CoV-2 infection. The specificity of these assays is relatively high. However, problems such as relatively low sensitivity, except for ELISA, and high false positive rates are present ${ }^{[73,74]}$. Immunoassays have several advantages: fast detection speed, easy operation, low cost, and easy promotion. However, the main limitation of these assays is their inability to separate NAbs from non-NAbs. Several VNTs have been developed for SARS-CoV-2; some were produced using live viruses ${ }^{[75]}$ or pseudotyped viruses expressing the S protein of SARS-CoV-2 ${ }^{[76-78]}$. Conventional VNT uses live cells expressing ACE2 and live SARS-CoV-2 virus ${ }^{[41]}$ and must be performed in a BSL-3 laboratory, in addition to relying on laborious microscopic counting of viral plaques by qualified personnel ${ }^{[79-81]}$. Several VNTs using pseudoviruses ${ }^{[77,78,82-85]}$ have been developed and evaluated to replace the traditional VNT using live virus ${ }^{[86]}$ in order to avoid the need for BSL-3 facilities and to speed up laboratory turnaround times ${ }^{[87-91]}$. Although the assays based on pseudovirus do not require BSL-3 containments ${ }^{[78]}$, they involve complex procedures that can be performed in several steps $^{[92]}$. In these assays, the ability to analyze NAbs is closely related to the maturity or titer of the virus as well as the type of cell lines and cell conditions used in the assay ${ }^{[93]}$. Thus, if the virus and host cells are not under optimal assay conditions, poor reproducibility can be generated ${ }^{[93]}$. In addition, poor correlation between the assays is not uncommon ${ }^{[94]}$.

\subsection{Plaque Reduction Neutralization Test}

In 1959, Henderson and Taylor developed the PRNT to detect arbovirus plaques and to measure the neutralization titer of serum Abs. Generally, PRNT is accepted as the most specific test among conventional serological tests. Although this method remains the gold standard for serological testing and determination of immune protection ${ }^{[95,96]}$, it requires working under strict biological containment conditions in BSL-3 laboratories when dealing with highly pathogenic $\mathrm{CoVs}^{[97]}$. Moreover, due to its low throughput, PRNT is not suitable for serodiagnosis or even large-scale vaccine evaluation. As with other CoVs outbreaks prior to the COVID-19 pandemic, PRNT has been described as a test for the SARS-CoV-2 NAbs ${ }^{[68,98]}$. PRNT can detect and quantify NAbs, both monoclonal Abs and polyclonal Abs, by determining the percentage of reduction in the viral activity since the viral concentration is generally constant. Alternatively, PRNT can be used as an alternative means to determine the level of protection by measuring the levels of binding of the virus-NAb complex. Under this context, NAbs can be served as a surrogate for protection against disease $^{[7]}$. In vaccine efficacy evaluation studies, PRNT is used to determine the protective efficacy of vaccine candidates by measuring the level of NAbs.

PRNT is basically designed to allow the formation of virus-Ab complexes in vitro and to measure the neutralizing effect of plaque formation on virussusceptible cells ${ }^{[7]}$. The virus-Ab complexes are formed by mixing SARS-CoV-2 viruses and the samples (e.g., serum or plasma) under well-defined incubation conditions and times. The virus-Ab complexes are then added to virus-susceptible cells, which are then covered with semi-solid medium (SSM) and incubated for 3-5 days, or until the cytopathic effect (CPE) and plaque formation are observed. SSM prevents the spread of viral progenitors in the medium, resulting in localized 
areas of CPE (plaques). Plaques are then revealed by adding dyes to the overlay or by direct staining of live cells after removing the overlay medium. However, the previous method increases the visible plaques compared to the conventional PRNT method. In conventional processes, dyes are usually added at the first or second overlay to monitor the development of plaques in live cells. Adding dyes after the removal of the overlay has many benefits. First, plaques do not need to be counted immediately, and chemically fixed cells can be stored stably for a long time ${ }^{[7]}$. Next, the titer of NAbs is determined by calculating and comparing the number of plaques with a standardized number of viruses to calculate the percentage of reduction in total viral infectivity. In samples containing SARS-CoV-2 specific NAbs, the number of plaques will be lower than in control wells because NAbs prevent infection of host cells. In the absence of specific SARS-CoV-2 NAbs, the number of plaques will be the same as those in control wells. This assay is tedious and laborious and requires BSL-3 working conditions. The presence of the SARS-CoV-2 virus is crucial in the assay used to measure NAbs against SARS-CoV-2 ${ }^{[41]}$. The advantage of the PRNT is that it reflects the level of the true live virus-NAb complexes. Indeed, the formation of the virusNAb complexes plays a central role in the protection against infection. During the interaction between viruses and NAbs, NAbs can prevent viruses from entering cells. Therefore, PRNT, although cumbersome and not suitable for routine testing of large samples, remains the gold standard for assessing $\mathrm{Ab}$-mediated neutralizing activity during SARS-CoV-2 infection.

\subsection{Pseudovirus-Based Neutralization Assay}

The pseudovirus-based neutralization assay (PBNA) has been shown to be more reliable than neutralization tests based on real live infectious viruses. Indeed, PBNA can be easily performed under BSL-2 conditions because pseudotyped viruses (PTVs) can only replicate for one cycle after entering cells. In contrast to other indirect assays, the PBNA can detect NAbs and inhibitors of viral entry and predict their function and efficacy. In addition, all PTVs contain reporter genes, which are usually expressed after infection to facilitate detection. Quantitative detection of NAbs is realized by recording data objectively with a luminometer (or flow cytometer), which puts PBNA in a highthroughput assay and makes it easy to standardize. However, PBNA can only evaluate NAbs of S protein, not other structural or non-structural proteins. Whether these proteins can induce NAbs is controversial ${ }^{[99-101]}$.

\subsubsection{Construction Methods of PTVs Packaging} Systems PTVs are viruses without pathogenicity and are designed to express the wild-type $\mathrm{S}$ protein of SARS-CoV-2 or its mutations with a C-terminal 18 or 19 amino-acid deletion. Specific molecules that contribute to signal readouts, such as GFP and luciferase, are often co-engineered into $\mathrm{PTVS}^{[102]}$. For example, PTVs were established based on vesicular stomatitis virus (VSV) to express the $\mathrm{S}$ protein ${ }^{[103]}$, as well as luciferase or enhanced GFP (eGFP) instead of VSV-G in the VSV genome ${ }^{[77,82,104]}$. Typically, three types of packaging systems are used to produce PTVs: lentiviral vectors (LVs), VSV, and murine leukemia virus packaging systems. Due to their high efficiency, LVs packaging systems are often the first choice to develop most PTVs envelopes. LVs were developed from lentiviruses such as feline immunodeficiency virus, simian immunodeficiency virus, and human immunodeficiency virus type 1 $(\mathrm{HIV}-1)^{[105]}$. As one of the retrovirus subfamilies, LVs share many characteristics with retroviruses, such as an RNA genome with the gag, pol, and env genes, which encode internal structural proteins (capsid), viral enzymes (reverse transcriptase and integrase), and envelope glycoproteins, respectively ${ }^{[106]}$. Among LVs, HIV was the first to be developed and are widely used in scientific research ${ }^{[107,108]}$. LVs carrying the HIV-1 backbone is an essential tool that offers several advantages compared to other viral vector systems ${ }^{[109]}$. Indeed, they are known to be a suitable promising gene delivery vehicle, as they can accommodate a large amount of genetic material ( 8 to $9 \mathrm{~kb}$ ), which extends their application. Indeed, it has been shown that most of the genes that are transferred can be cloned into $\mathrm{LVs}^{[110]}$. LVs can also infect a wide variety of dividing and non-dividing cells and support the long-term transgene expression through stable integration into the host genome ${ }^{[111-114]}$. In addition, a wide variety of glycoproteins can be used to form PTVs with LVs, increasing their utility. Compared to other vectors, LVs allow rapid expression of the transgene ${ }^{[115]}$ and exhibit minimal immunogenicity and toxicity ${ }^{[16]}$. Although LVs have some great advantages, there are also some drawbacks. Indeed, based on HIV-1, recombinant LVs require at least three HIV-1 genes ( $\mathrm{gag}$, pol, and rev) for production (table 1).

3.2.2 Structure of Lentiviral Vectors The tropism of lentiviruses is determined by the interaction of viral surface glycoproteins with receptor molecules expressed on the cell surface and can be altered by

Table 1 Advantages and disadvantages of LVs packaging system 
incorporating heterologous glycoproteins derived from other enveloped viruses ${ }^{[117]}$. The technique described here is called pseudotyping, which can be used to achieve selective entry into specific target cells by taking advantage of protein's heterologous envelope and natural tropism. Generally, viral vectors are combined from different plasmids, which can be divided into two parts. The first part containing the packaging system is required for the formation and infectivity of the viral particles, and the second part containing the transfer vector includes cis-acting sequences sufficient to mobilize the viral genome. Advances in LVs production have led to the development of replication-incompetent LVs, which can be produced easily with minimal effort. Replication-incompetent LVs are produced by replacing all viral genes in the lentivirus genome with a transgene of interest, leaving only the cis-acting elements essential for viral packaging. Components removed from the viral genome are supplied in trans by separate plasmids encoding the viral genes required for packaging and envelope pseudotyping. The transfer vector containing the transgene of interest and the separated helper plasmids is co-transfected into a mammalian cell line. The LVs can be harvested and purified from the medium. Providing the necessary viral genes in separate plasmids greatly reduces the possibility of mobilization and producing a competent replication virus. This system also provides the ability to easily mix and match glycoproteins from alternative enveloped viruses to optimize LVs expression levels and tropism specifically for the desired application ${ }^{[118,119]}$. Several packaging systems are used to create PTVs, with lentivirus-based vectors often used ${ }^{[120]}$. These lentiviral PTVs systems, particularly the use of vectors derived from HIV-1, have been used to investigate novel $\mathrm{CoVs}$ and their cell tropisms under conventional biosafety conditions ${ }^{[121-124]}$. HIV-1 based vectors are used as examples to describe the structure of LVs. Vector safety is a key issue in vector design, and given the pathogenicity of the parental virus, in the case of HIV-1 based vectors, several biosecurity concerns are heightened. The design of HIV-1-based vectors has progressed through a number of socalled generations ${ }^{[125]}$. There are three generations of LVs. The first and second generations of LVs consist of three plasmids, while the third consists of four ${ }^{[108]}$. Traditionally, LVs particles are generated by co-transfection of three plasmids into HEK 293T cell lines ${ }^{[126]}$. This common procedure of separating constructs into three plasmids reduces the possibility of recombination into a competent replication virus. The three plasmids consist of a packaging plasmid, a transfer plasmid, and an envelope encoding plasmid.

3.2.3 First Generation of Recombinant Lentiviral Vectors To reduce the risk of producing replicationcompetent lentiviruses, the first generation recombinant
LVs based on HIV-1 divide the HIV genome into three separate plasmids: the packaging plasmid, which contains gag and pol sequences, the viral regulatory genes tat and rev, and the accessory genes vif, vpr, $v p u$, and nef; an envelope plasmid expressing a viral glycoprotein; and a transfer plasmid, which encodes proteins necessary for reverse transcription, packaging, and integration but not for the expression of the HIV protein $^{[127]}$. This separation of the genomic components responsible for packaging viral DNA from the genomic components that activate them prevents the packaging sequences from incorporating into the viral genome, consequently preventing the virus from reproducing after infection of a host cel[ ${ }^{[128]}$.

\subsubsection{Second Generation of Recombinant Lentiviral} Vectors Despite these safety measures, the firstgeneration recombinant LVs still pose a biosecurity risk due to the viral accessory proteins vif, vpu, vpr, and nef. Although these viral accessory proteins are important for HIV replication in primary cells, they are not essential for lentiviral function, prompting the development of second-generation recombinant LVs where they have been eliminated ${ }^{[129]}$. Thus, secondgeneration systems only had 4 ( $\mathrm{gag}, \mathrm{pol}$, rev, and tat) of 9 genes in the HIV backbone ${ }^{[130]}$. Despite these changes, the second-generation lentivirus system is not without its flaws due to the existence of long terminal repeats (LTRs). These LTRs flank the transgene cassette, which is integrated into the host genome ${ }^{[131]}$. However, their existence can give rise to potential dangers. Recombination events leading to the production of replication-competent lentiviruses can allow these viruses to replicate like wild-type viruses, as LTRs also have a promoter region and a binding site for host transcription factors. Integrating LTRs into the host genome can activate adjacent cellular genes that may be proto-oncogenes ${ }^{[131]}$. By identifying unnecessary genes for the therapeutic gene(s) transfer, second-generation packaging systems have been developed where vif, vpr, $v p u$, and nef have been removed ${ }^{[130]}$ with the primary objective of dramatically improving the safety profile of LVs.

\subsubsection{Third Generation of Recombinant Lentiviral}

Vectors The packaging system of the third generation of LVs involves four plasmids, which provide the necessary trans action factors, namely gag-pol, rev, and an envelope protein, respectively. Third-generation recombinant self-inactivating LVs have been developed to improve the safety of second-generation systems. These third-generation systems have the U3 region of 3'-LTR deleted. This deletion is then copied into the $5^{\prime}$-LTR promoter and enhancer region of the integrated genome in reverse transcription, resulting in the transcriptional inactivation of the potentially packable viral genome in the host cell ${ }^{[132]}$. Second, the packaging plasmid is split into two separate plasmids, 
incorporating rev and the other incorporating gag and pol. The tat and rev genes are necessary for HIV-1 replication because they regulate viral transcription and nuclear export of transcripts ${ }^{[133]}$. To reduce the risk of replication, the formation of competent lentiviruses is suppressed in the third-generation packaging plasmid, and its function is replaced by a constitutively active promoter sequence independent of tat ${ }^{[134]}$. The tat gene is essential for viral replication; thus, by its elimination, LVs biosafety is further enhanced. Dividing the thirdgeneration vector system into four separate plasmids decreases the chances of formation of replicationcompetent HIV-1 type viruses because at least three recombination events would be required. However, these safety improvements could come at a cost. Compared to the second-generation plasmid system, virus yield may be lower ${ }^{[135]}$.

3.2.6 Reporter Genes After creating PTVs and their subsequent inoculation into a cell line, it is essential to determine whether viral entry and transduction from a cell have occurred. Different reporter genes, generally located on the transfer plasmid, allow visualization and quantification of this event. Luciferase is an enzyme commonly used as a reporter gene. After PVTs entry and cell transduction, luciferase is produced, allowing viral entry to be quantified by lysing cells, releasing luciferase, and allowing it to catalyze a chemical reaction in which luciferin is converted to oxyluciferin with light released as a by-product ${ }^{[136]}$. The enzymatic activity of luciferase can then be measured using a luminometer. Green fluorescent protein (GFP) is another commonly used reporter gene. GFP fluoresces bright green upon exposure to blue light (450-490 nm), allowing it to be detected by fluorescent microscopy and quantified by manual cell counting or fluorescenceactivated cell sorting ${ }^{[137]}$. GFP can be expressed by the cell without a cytotoxic effect and can be visualized in living cells, making it ideal for monitoring the cellular entry of PTVs into cell lines.

3.2.7 Quantification of Lentiviral Vectors Reliable methods for quantifying LVs are needed to support process development and enable clinical trial material quality control. The four broad categories of available techniques for virus quantification are (1) determining the titer of infectious particles, (2) measuring the presence or function of viral proteins, (3) detecting the presence of the marker or viral nucleic acid in the viral genome, and (4) enumerating the physical viral particles, whether labeled or unlabeled ${ }^{[138]}$. The quantity of infectious particles in an LVs preparation reported as the number of transduction-competent lentivirus particles per $\mathrm{mL}$ of virus stock is determined using the limiting dilution of the vector, followed by transduction of the target cells and measurement of the expression of the marker using flow cytometry ${ }^{[139]}$. The most commonly used reporter protein is eGFP, which allows straightforward analysis of functional titers by flow cytometry. This method, however, does not distinguish between single and multiple vector integrations. These pseudovirus-based VNTs were developed to avoid the use of real live SARS-CoV-2 viruses ${ }^{[78,82-85,104]}$. These tests only require BSL- 1 or -2 facilities ${ }^{[83]}$, which are more accessible to biomedical researchers. Although the evaluation of NAbs against SARS-CoV-2 in the clinical setting is critical ${ }^{[65,83,90,104,140,141]}$, the ability to perform VNT in BSL-1 or 2 laboratories is preferred. When PTVs are incubated with samples containing NAbs or molecular inhibitors before cell infection, they will be able to block virus entry into cells. The number of blocking viruses can be assessed by the reduction of chemiluminescence or fluorescent cells, reflecting the level of NAbs or molecular inhibitors in the sample. The development of PRNTs provides an opportunity to pre-screen and restrict PRNT to samples with a high probability of obtaining better titers of NAbs.

\subsection{Enzyme-Linked Immunosorbent Assay}

ELISAs are standard-essential tools in the biomedical field. ELISA is a term used to describe any solid phase immuno-enzymatic panel test that uses enzymatic chemiluminescence to identify the presence and concentration of an $\mathrm{Ag}$ or $\mathrm{Ab}$ in liquid sample. The fact that these tests use Ags/Abs labeled with enzymes makes it possible to detect specific molecules in the samples. Likewise, these tests are characterized by their simplicity and economy of operation ${ }^{[142]}$. The color change can be measured using a spectrophotometer combined with computer software, which can further calculate data and display results. ELISA is a popular and routine immunoassay used in many biomedical, clinical, and immunological laboratories. According to standard procedures, it is also possible to perform ELISA on a large scale with a robotic liquid handling workstation. Typically, the basic steps of ELISA include sequential coating, blocking, washing, signal generation, and plate reading. There are four frequently used iterations of this assay: indirect, direct, sandwich, and competition, with direct being the most commonly used. To choose an appropriate ELISA iteration, several necessary factors must be considered, such as the number of candidate Abs, the Ag of interest, the sensitivity range, the binding capacity of solid-phase, and even the $\mathrm{pH}$ of coating buffer.

With the current COVID-19 pandemic, several ELISAs have been developed and made available for the detection and quantification of SARS-CoV-2

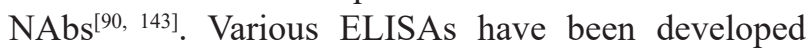
using recombinant RBD protein or ACE2 as the coating Ag, and horseradish peroxidase (HRP) labeled ACE2 or RBD labeled HRP to produce a detection signal. These ELISAs can be performed within a few hours in a BSL-1 or 2 containment. Recently, a new, simpler, and faster ELISA capable of detecting SARS- 
CoV-2 NAbs based on ACE2-RBD interactions has been reported ${ }^{[144]}$. This assay mimics the virus-host interaction in an ELISA and can be performed in a BSL2 laboratory within a few hours. Many investigations have utilized the ELISA method to study the timeline or chronology of $\mathrm{Ab}$ responses in COVID-19 patients or in vaccinated individuals ${ }^{[145]}$. To combat the SARS-CoV-2 virus, Schöler et al ${ }^{[146]}$ established a neutralization test for SARS-CoV-2 using an in-cell ELISA approach (icELISA). Indeed, this assay allows rapid quantification ( $<48 \mathrm{~h}$ in total, readings in seconds) and automation of COVID-19 infection in cell culture, using reagents and installations present in most routine diagnostic services to evaluate the effectiveness of NAbs and antiviral drugs ${ }^{[146]}$.

\subsection{Lateral Flow Immunoassay}

The lateral flow immunoassay (LFIA) is among several well-known immunoassays. This assay is fast and practical in terms of use and the acquisition of results. LFIA is a qualitative testing device usually applied in detection of SARS-CoV-2 Abs within 5-30 minutes in serum, plasma, whole blood, and other fluids ${ }^{[147]}$, can be performed in a professional laboratory or by an individual, and are applicable in hospitals, emergency rooms, and other patient care settings ${ }^{[148]}$. Therefore, LFIA should be used as a necessary adjunct to all available neutralization tests. LFIA, also known as a lateral flow immunochromatographic assay, is a simple device developed based on the point-of-care testing strategy. LFIA can easily detect the presence of a specific analyte in body fluids. Structurally, each LFIA is based on a variety of hair materials. Each segment is overlappingly mounted on a support card that spontaneously delivers the liquid sample by capillary action. Typically, the sample pad is at the proximal end of the assay cartridge that can be soaked with sufficient sample. The soaked liquid sample enters a second zone, the conjugate pad, which contains a dry conjugate that captures the target analytes in the migrating liquid sample. The analyte-specific $\mathrm{Ab}$ or Ag is still used as this bioactive conjugate and is covalently linked to colloidal gold as the conjugate typically; however, other materials can also be applied in place of colloidal gold, such as colored or paramagnetic monodisperse latex particles ${ }^{[147,149]}$. The analyte-conjugate-target particles then flow to the third zone, which is made of porous material. A detection bar and a control bar are usually immobilized separately, with a certain distance between them in this zone. The former is designed to capture the analyte, so that analyte-binding particles can be enriched. At the same time, the latter band is used as a control to ensure that the test is working well. When the analyte-conjugate particles reach these bands, i.e., where the $\mathrm{Ag}$ or $\mathrm{Ab}$ is immobilized, it captures the complexes. When more complexes accumulate on the capture band line, the results can be observed directly and visually or with a signal detector. Meanwhile, the absorbent pad can absorb liquid after excess liquid has passed through the capture band. LFIA performance can be affected by many factors, such as material, sample viscosity, $\mathrm{Ab}$ affinity, signal generation system, etc. Due to its low cost and high availability, it can be widely used to detect and diagnose various Ags and Abs associated with any disease. However, although these LFIAs have proven convenient for testing and useful in laboratories with limited resources, their clinical performance is limited and characterized by various sensitivities and specificities $^{[150-152]}$. One meta-analysis showed that the combined sensitivity of LFIAs for COVID-19 was only $66 \%$, far behind the combined sensitivity of ELISA, which was $84.3 \%{ }^{[153]}$. In the fight against this COVID-19 pandemic, several LFIAs are now available on the market. In a recent study, Yun et al reported that five LFIAs (Boditech Med, SD Biosensor, PCL, Sugentech, and Rapigen) showed reliable performance with a percent positive agreement ranging from $84.0 \%$ to $98.5 \%$ on samples tested more than 14 days after symptom onset ${ }^{[154]}$. Li and colleagues designed another study to detect the IgM and IgG Abs in blood samples of 397 clinically positive and 128 clinically negative COVID-19 patients using the IgM/IgG combined $\mathrm{Ab}$ test. They reported $88.66 \%$ sensitivity and $90.63 \%$ specificity for overall testing. Additionally, they found that $\operatorname{IgM}$ and $\operatorname{IgG}$ Abs were positive in $64.48 \%$ of patients $^{[155]}$.

\section{CONCLUSION}

In summary, each neutralization test has advantages and disadvantages (fig. 3). ELISAs are widely used in commercial tests and can be easily performed in routine diagnostic laboratories and can test a large number of samples. The two virus neutralization tests require cell culture facilities, with PRNT necessitating access to BSL- 3 facilities. In contrast, the pseudovirus neutralization assay can be adapted for high-throughput screening without the need for a BSL-3 facility.

\section{Conflict of Interest Statement}

We declare that we have no conflicts of interest.

\section{REFERENCES}

1 Weekly epidemiological update on COVID-19 - 21 September 2021. Available from: https://www.who.int/ publications $/ \mathrm{m} /$ item/weekly-epidemiological-updateon-covid-19-21-september-2021. [Accessed Sep 22, 2021].

2 Tang Q, Song Y, Shi M, et al. Inferring the hosts of coronavirus using dual statistical models based on nucleotide composition. Sci Rep, 2015,5:17155

3 Cui J, Li F, Shi ZL. Origin and evolution of pathogenic coronaviruses. Nat Rev Microbiol, 2019,17(3):181-192 


\section{Advantages}

Plaque reduction neutralization test (PRNT):

It's the main gold standard for serological testing and determining immune protection by giving high level of the real live virus-NAbs complex.

Pseudovirus based neutralization assay (PBNA)

a) Much easier to handle (do not require BSL-3 laboratories)

b) Can detect weak antibody responses and allow higher throughput

c) More objective and less labor intensive

d) Versatile and save more time

e) Detect NAbs and inhibitors of viral entry

f) Predict NAbs function and efficacy

Enzyme-linked immunosorbent assay (ELISA):

a) Relative high specificity

b) Fast detection speed, easy operation, low cost, and easy promotion

c) Convenient to compare multiple samples from a single patient

d) Suitable for routine testing of large samples

Lateral flow immunoassay (LFIA)

a) Fast (Relatively short development time 5-30 min and uses visual detection by the naked eye)

b) Low cost, low sample volume

c) Simple assay procedure

d) Application at point of care

e) Can detect and diagnose various Ags and Abs associated with any disease
Disadvantages

Plaque reduction neutralization test (PRNT):

a) Require the use of live viruses and a biosafety level 3 (BSL-3) containment facility and are not suitable for routine testing of large samples

b) Use microscopy counting

c) Time consuming

d) Require a qualified personal

Pseudovirus based neutralization assay (PBNA):

- Can only evaluate NAbs for S protein and not other structural or non-structural proteins

Enzyme-linked immunosorbent assay (ELISA):

a) High rate of false positives

b) Inability to separate NAbs from non-NAbs

Lateral flow immunoassay (LFIA):

a) Restriction on total volume in test gives a limit of sensitivity and specificities

b) Inaccurate sample volume reduces precision

c) Excellent antibody preparation is obligatory

d) Analysis time is dependent on nature of sample (Viscosity)

e) Antibody affinity

Fig. 3 Advantages and disadvantages of SARS-CoV-2 NAbs detection methods

4 Chan-Yeung $M$, Xu RH. SARS: epidemiology. Respirology, 2003,8(Suppl):S9-S14

5 Middle East respiratory syndrome coronavirus (MERS-CoV). Available from: https://www.who.int/ westernpacific/health-topics/middle-east-respiratorysyndrome-coronavirus-mers. [Accessed September 13, 2021].

6 Chan JFW, Yuan S, Kok KH, et al. A familial cluster of pneumonia associated with the 2019 novel coronavirus indicating person-to-person transmission: a study of a family cluster. Lancet, 2020, 395(10223):514-523

7 Kampf G, Todt D, Pfaender S, et al. Persistence of coronaviruses on inanimate surfaces and their inactivation with biocidal agents. J Hosp Infect, 2020, 104(3):246-251

$8 \mathrm{Wu}$ Z, McGoogan JM. Characteristics of and Important Lessons From the Coronavirus Disease 2019 (COVID-19) Outbreak in China: Summary of a Report of 72314 Cases From the Chinese Center for Disease Control and Prevention. JAMA, 2020,323(13):12391242

9 Yu C, Lei Q, Li W, et al. Clinical Characteristics, Associated Factors, and Predicting COVID-19 Mortality Risk: A Retrospective Study in Wuhan, China. Am J Prev Med, 2020,59(2):168-175

10 Coronaviridae Study Group of the International Committee on Taxonomy of Viruses. The species Severe acute respiratory syndrome-related coronavirus: classifying 2019-nCoV and naming it SARS-CoV-2. Nat Microbiol, 2020,5(4):536-544

11 Chen Y, Liu Q, Guo D. Emerging coronaviruses: Genome structure, replication, and pathogenesis. J Med Virol, 2020,92(10):2249

12 Li C, Yang Y, Ren L. Genetic evolution analysis of 2019 novel coronavirus and coronavirus from other species. Infect Genet Evol, 2020,82:104285

13 Wu A, Peng Y, Huang B, et al. Genome Composition and Divergence of the Novel Coronavirus (2019$\mathrm{nCoV})$ Originating in China. Cell Host Microbe, 2020,27(3):325-328

14 Zhu N, Zhang D, Wang W, et al. A Novel Coronavirus from Patients with Pneumonia in China, 2019. N Engl J Med, 2020,382(8):727-733

15 Wang C, Liu Z, Chen Z, et al. The establishment of reference sequence for SARS-CoV-2 and variation analysis. J Med Virol, 2020,92(6):667-674

16 Yoshimoto FK. The Proteins of Severe Acute Respiratory Syndrome Coronavirus-2 (SARS CoV-2 or n-COV19), the Cause of COVID-19. Protein J, 2020,39(3):198-216

17 Chan JFW, Kok KH, Zhu Z, et al. Genomic characterization of the 2019 novel human-pathogenic coronavirus isolated from a patient with atypical pneumonia after visiting Wuhan. Emerg Microbes Infect, 2020,9(1):221-236

18 Vashist SK. In Vitro Diagnostic Assays for COVID-19: Recent Advances and Emerging Trends. Diagnostics (Basel), 2020,10(4):E202

19 Wrapp D, Wang N, Corbett KS, et al. Cryo-EM structure of the 2019-nCoV spike in the prefusion conformation. Science, 2020,367(6483):1260-1263

20 Walls AC, Park Y-J, Tortorici MA, et al. Structure, Function, and Antigenicity of the SARS-CoV-2 Spike Glycoprotein. Cell, 2020,183(6):1735

21 Huang Y, Yang C, Xu X-F, et al. Structural and functional properties of SARS-CoV-2 spike protein: potential antivirus drug development for COVID-19. Acta Pharmacol Sin, 2020,41(9):1141-1149

22 Premkumar L, Segovia-Chumbez B, Jadi R, et al. The 
receptor binding domain of the viral spike protein is an immunodominant and highly specific target of antibodies in SARS-CoV-2 patients. Sci Immunol, 2020,5(48):eabc8413

23 Dan JM, Mateus J, Kato Y, et al. Immunological memory to SARS-CoV-2 assessed for up to 8 months after infection. Science, 2021,371(6529):eabf4063

24 To KKW, Tsang OTY, Leung WS, et al. Temporal profiles of viral load in posterior oropharyngeal saliva samples and serum antibody responses during infection by SARS-CoV-2: an observational cohort study. Lancet Infect Dis, 2020,20(5):565-574

25 Iyer AS, Jones FK, Nodoushani A, et al. Persistence and decay of human antibody responses to the receptor binding domain of SARS-CoV-2 spike protein in COVID-19 patients. Sci Immunol, 2020,5(52):eabe0367

26 Röltgen K, Powell AE, Wirz OF, et al. Defining the features and duration of antibody responses to SARS$\mathrm{CoV}-2$ infection associated with disease severity and outcome. Sci Immunol, 2020, 5(54):eabe0240

27 Seow J, Graham C, Merrick B, et al. Longitudinal observation and decline of neutralizing antibody responses in the three months following SARS-CoV-2 infection in humans. Nat Microbiol, 2020,5(12):15981607

28 Tang F, Quan Y, Xin ZT, et al. Lack of peripheral memory B cell responses in recovered patients with severe acute respiratory syndrome: a six-year follow-up study. J Immunol, 2011,186(12):7264-7268

29 Ibarrondo FJ, Fulcher JA, Goodman-Meza D, et al. Rapid Decay of Anti-SARS-CoV-2 Antibodies in Persons with Mild Covid-19. N Engl J Med, 2020,383(11):1085-1087

30 Gudbjartsson DF, Norddahl GL, Melsted P, et al. Humoral Immune Response to SARS-CoV-2 in Iceland. N Engl J Med, 2020,383(18):1724-1734

31 Ripperger TJ, Uhrlaub JL, Watanabe M, et al. Orthogonal SARS-CoV-2 Serological Assays Enable Surveillance of Low-Prevalence Communities and Reveal Durable Humoral Immunity, Immunity, 2020,53(5):925-933.e4.

32 Hsueh PR, Huang LM, Chen PJ, et al. Chronological evolution of IgM, IgA, IgG and neutralisation antibodies after infection with SARS-associated coronavirus. Clin Microbiol Infect, 2004,10(12):1062-1066

33 He Z, Dong Q, Zhuang H, et al. Kinetics of severe acute respiratory syndrome (SARS) coronavirus-specific antibodies in 271 laboratory-confirmed cases of SARS. Clin Diagn Lab Immunol, 2004,11(4):792-794

34 Zumla A, Hui DS, Perlman S. Middle East respiratory syndrome. Lancet, 2015, 386(9997):995-1007

35 Edridge AWD, Kaczorowska J, Hoste ACR, et al. Seasonal coronavirus protective immunity is shortlasting. Nat Med, 2020,26(11):1691-1693

36 Callow KA, Parry HF, Sergeant M, et al. The time course of the immune response to experimental coronavirus infection of man. Epidemiol Infect, 1990,105(2):435446

37 Choe PG, Perera R a. PM, Park WB, et al. MERS-CoV Antibody Responses 1 Year after Symptom Onset, South Korea, 2015. Emerg Infect Dis, 2017,23(7):1079-1084

38 Gasser R, Cloutier M, Prévost J, et al. Major role of IgM in the neutralizing activity of convalescent plasma against SARS-CoV-2. Cell Rep, 2021,34(9):108790
39 Zhao J, Yuan Q, Wang H, et al. Antibody Responses to SARS-CoV-2 in Patients With Novel Coronavirus Disease 2019. Clin Infect Dis, 2020,71(16):2027-2034

40 Long Q-X, Liu BZ, Deng HJ, et al. Antibody responses to SARS-CoV-2 in patients with COVID-19. Nat Med, 2020,26(6):845-848

41 Suthar MS, Zimmerman MG, Kauffman RC, et al. Rapid Generation of Neutralizing Antibody Responses in COVID-19 Patients. Cell Rep Med, 2020,1(3):100040

$42 \mathrm{Ma} \mathrm{H}$, Zeng W, He H, et al. Serum IgA, IgM, and IgG responses in COVID-19. Cell Mol Immunol, 2020, 17(7):773-775

43 Kellam P, Barclay W. The dynamics of humoral immune responses following SARS-CoV-2 infection and the potential for reinfection. J Gen Virol. 2020,101(8):791797

44 Du L, He Y, Zhou Y, et al. The spike protein of SARSCoV--a target for vaccine and therapeutic development. Nat Rev Microbiol, 2009,7(3):226-236

45 Li F. Structure, Function, and Evolution of Coronavirus Spike Proteins. Annu Rev Virol, 2016,3(1):237-261

46 Cho H, Excler JL, Kim JH, et al. Development of Middle East Respiratory Syndrome Coronavirus vaccines advances and challenges. Hum Vaccin Immunother, 2018,14(2):304-313

47 Matsuyama S, Nao N, Shirato K, et al. Enhanced isolation of SARS-CoV-2 by TMPRSS2-expressing cells. Proc Natl Acad Sci USA, 2020,117(13):70017003

48 Zhu Z, Chakraborti S, He Y, et al. Potent cross-reactive neutralization of SARS coronavirus isolates by human monoclonal antibodies. Proc Natl Acad Sci USA. 2007,104(29):12123-12128

49 Rockx B, Corti D, Donaldson E, et al. Structural basis for potent cross-neutralizing human monoclonal antibody protection against lethal human and zoonotic severe acute respiratory syndrome coronavirus challenge. J Virol, 2008,82(7):3220-3235

50 Du L, Yang Y, Zhou Y, et al. MERS-CoV spike protein: a key target for antivirals. Expert Opin Ther Targets, 2017,21(2):131-143

51 Zhou Y, Yang Y, Huang J, et al. Advances in MERS$\mathrm{CoV}$ Vaccines and Therapeutics Based on the ReceptorBinding Domain. Viruses, 2019,11(1):E60

52 Poh CM, Carissimo G, Wang B, et al. Potent neutralizing antibodies in the sera of convalescent COVID-19 patients are directed against conserved linear epitopes on the SARS-CoV-2 spike protein. bioRxiv, 2020:15461

53 Meyer B, Drosten C, Müller MA. Serological assays for emerging coronaviruses: challenges and pitfalls. Virus Res, 2014,194:175-183

54 Yan R, Zhang Y, Li Y, et al. Structural basis for the recognition of SARS-CoV-2 by full-length human ACE2. Science. 2020,367(6485):1444-1448

55 Baden LR, El Sahly HM, Essink B, et al. Efficacy and Safety of the mRNA-1273 SARS-CoV-2 Vaccine. N Engl J Med, 2021,384(5):403-416

56 Polack FP, Thomas SJ, Kitchin N, et al. Safety and Efficacy of the BNT162b2 mRNA Covid-19 Vaccine. N Engl J Med, 2020,383(27):2603-2615

57 Li Y, Ma ML, Lei Q, et al. Linear epitope landscape of the SARS-CoV-2 Spike protein constructed from 1,051 
COVID-19 patients. Cell Rep, 2021,34(13):108 915

58 Piccoli L, Park YJ, Tortorici MA, et al. Mapping Neutralizing and Immunodominant Sites on the SARSCoV-2 Spike Receptor-Binding Domain by StructureGuided High-Resolution Serology. Cell, 2020,183(4): 1024-1042.e21

59 Addetia A, Crawford KHD, Dingens A, et al. Neutralizing Antibodies Correlate with Protection from SARS-CoV-2 in Humans during a Fishery Vessel Outbreak with a High Attack Rate. J Clin Microbiol, 2020,58(11):e02107-e02120

60 Moore JP, Klasse PJ. COVID-19 Vaccines: "Warp Speed" Needs Mind Melds, Not Warped Minds. J Virol, 2020,94(17):e01083-e01020

61 Zohar T, Alter G. Dissecting antibody-mediated protection against SARS-CoV-2. Nat Rev Immunol, 2020,20(7):392-394

62 Zost SJ, Gilchuk P, Case JB, et al. Potently neutralizing and protective human antibodies against SARS-CoV-2. Nature, 2020,584(7821):443-449

63 Lei Q, Hou H, Yu C, et al. Kinetics of Neutralizing Antibody Response Underscores Clinical COVID-19 Progression. J Immunol Res, 2021,2021:9822706

64 Cao Y, Su B, Guo X, et al. Potent Neutralizing Antibodies against SARS-CoV-2 Identified by HighThroughput Single-Cell Sequencing of Convalescent Patients' B Cells. Cell, 2020,182(1):73-84.e16

65 Lei Q, Li Y, Hou HY, et al. Antibody dynamics to SARS-CoV-2 in asymptomatic COVID-19 infections. Allergy, 2021,76(2):551-561

66 Tani H, Kimura M, Tan L, et al. Evaluation of SARSCoV-2 neutralizing antibodies using a vesicular stomatitis virus possessing SARS-CoV-2 spike protein. Virol J, 2021,18:16

67 Bloch EM, Shoham S, Casadevall A, et al. Deployment of convalescent plasma for the prevention and treatment of COVID-19. J Clin Invest, 2020,130(6):2757-2765

68 Wölfel R, Corman VM, Guggemos W, et al. Virological assessment of hospitalized patients with COVID-2019. Nature, 2020,581(7809):465-469

69 Hoffmann M, Kleine-Weber H, Schroeder S, et al. SARS-CoV-2 Cell Entry Depends on ACE2 and TMPRSS2 and Is Blocked by a Clinically Proven Protease Inhibitor. Cell, 2020,181(2):271-280.e8

$70 \mathrm{Ou}$ X, Liu Y, Lei X, et al. Characterization of spike glycoprotein of SARS-CoV-2 on virus entry and its immune cross-reactivity with SARS-CoV. Nat Commun, 2020,11(1):1620

71 Poh CM, Carissimo G, Wang B, et al. Two linear epitopes on the SARS-CoV-2 spike protein that elicit neutralising antibodies in COVID-19 patients. Nat Commun, 2020,11(1):2806

72 Theel ES, Slev P, Wheeler S, et al. The Role of Antibody Testing for SARS-CoV-2: Is There One? J Clin Microbiol, 2020,58(8):e00797-20

73 Lester S, Harcourt J, Whitt M, et al. Middle East respiratory coronavirus (MERS-CoV) spike (S) protein vesicular stomatitis virus pseudoparticle neutralization assays offer a reliable alternative to the conventional neutralization assay in human seroepidemiological studies. Access Microbiol, 2019,1(9):e000057

74 Degnah AA, Al-Amri SS, Hassan AM, et al.
Seroprevalence of MERS-CoV in healthy adults in western Saudi Arabia, 2011-2016. J Infect Public Health, 2020,13(5):697-703

75 Mendoza EJ, Manguiat K, Wood H, et al. Two Detailed Plaque Assay Protocols for the Quantification of Infectious SARS-CoV-2. Curr Protoc Microbiol, 2020, 57(1):cpmc105

$76 \mathrm{Ni}$ L, Ye F, Cheng ML, et al. Detection of SARS-CoV-2Specific Humoral and Cellular Immunity in COVID-19 Convalescent Individuals. Immunity, 2020,52(6):971977.e3

77 Nie J, Li Q, Wu J, et al. Establishment and validation of a pseudovirus neutralization assay for SARS-CoV-2. Emerg Microbes Infect, 2020,9(1):680-686

78 Crawford KHD, Eguia R, Dingens AS, et al. Protocol and Reagents for Pseudotyping Lentiviral Particles with SARS-CoV-2 Spike Protein for Neutralization Assays. Viruses, 2020,12(5):E513

79 Manenti A, Maggetti M, Casa E, et al. Evaluation of SARS-CoV-2 neutralizing antibodies using a CPEbased colorimetric live virus micro-neutralization assay in human serum samples. J Med Virol, 2020:92(10): 2096-2104

80 Okba NMA, Müller MA, Li W, et al. Severe Acute Respiratory Syndrome Coronavirus 2-Specific Antibody Responses in Coronavirus Disease Patients. Emerg Infect Dis, 2020,26(7):1478-1488

81 Perera RA, Mok CK, Tsang OT, et al. Serological assays for severe acute respiratory syndrome coronavirus 2 (SARS-CoV-2), March 2020. Euro Surveill, 2020, 25(16):2000421

82 Xiong HL, Wu YT, Cao JL, et al. Robust neutralization assay based on SARS-CoV-2 S-protein-bearing vesicular stomatitis virus (VSV) pseudovirus and ACE2-overexpressing BHK21 cells. Emerg Microbes Infect, 2020,9(1):2105-2113

83 Zettl F, Meister TL, Vollmer T, et al. Rapid Quantification of SARS-CoV-2-Neutralizing Antibodies Using Propagation-Defective Vesicular Stomatitis Virus Pseudotypes. Vaccines (Basel), 2020,8(3):E386

84 Schmidt F, Weisblum Y, Muecksch F, et al. Measuring SARS-CoV-2 neutralizing antibody activity using pseudotyped and chimeric viruses. J Exp Med, 2020, 217(11):e20201181

85 Xie X, Muruato AE, Zhang X, et al. A nanoluciferase SARS-CoV-2 for rapid neutralization testing and screening of anti-infective drugs for COVID-19. Nat Commun, 2020,11(1):5214

86 Focosi D, Maggi F, Mazzetti P, et al. Viral infection neutralization tests: A focus on severe acute respiratory syndrome-coronavirus-2 with implications for convalescent plasma therapy. Rev Med Virol, 2021,31(2):e2170

87 Rosado J, Pelleau S, Cockram C, et al. Multiplex assays for the identification of serological signatures of SARSCoV-2 infection: an antibody-based diagnostic and machine learning study. Lancet Microbe, 2021,2(2): e60-e69

88 Taylor SC, Hurst B, Charlton CL, et al. A New SARSCoV-2 Dual-Purpose Serology Test: Highly Accurate Infection Tracing and Neutralizing Antibody Response Detection. J Clin Microbiol, 2021,59(4):e02438-e02420 
89 Muruato AE, Fontes-Garfias CR, Ren P, et al. A highthroughput neutralizing antibody assay for COVID-19 diagnosis and vaccine evaluation. Nat Commun, 2020, 11(1):4059

90 Tan CW, Chia WN, Qin X, et al. A SARS-CoV-2 surrogate virus neutralization test based on antibodymediated blockage of ACE2-spike protein-protein interaction. Nat Biotechnol, 2020,38(9):1073-1078

91 Byrnes JR, Zhou XX, Lui I, et al. Competitive SARSCoV-2 Serology Reveals Most Antibodies Targeting the Spike Receptor-Binding Domain Compete for ACE2 Binding. mSphere, 2020,5(5):e00802-e00820

92 Ferrara F, Temperton N. Pseudotype Neutralization Assays: From Laboratory Bench to Data Analysis. Methods Protoc, 2018,1(1):8

93 Mukherjee S, Dowd KA, Manhart CJ, et al. Mechanism and significance of cell type-dependent neutralization of flaviviruses. J Virol, 2014,88(13):7210-7220

94 Liu L, Wang P, Nair MS, et al. Potent neutralizing antibodies against multiple epitopes on SARS-CoV-2 spike. Nature, 2020,584(7821):450-456

95 Perera RA, Mok CK, Tsang OT, et al. Serological assays for severe acute respiratory syndrome coronavirus 2 (SARS-CoV-2), March 2020. Euro Surveill, 2020,25(16): 200421

96 Okba NMA, Müller MA, Li W, et al. Severe Acute Respiratory Syndrome Coronavirus 2-Specific Antibody Responses in Coronavirus Disease Patients. Emerg Infect Dis, 2020,26(7):1478-1488

97 Algaissi A, Hashem AM. Evaluation of MERS$\mathrm{CoV}$ Neutralizing Antibodies in Sera Using Live Virus Microneutralization Assay. Methods Mol Biol, 2020,2099:107-116

98 Haveri A, Smura T, Kuivanen S, et al. Serological and molecular findings during SARS-CoV-2 infection: the first case study in Finland, January to February 2020. Euro Surveill, 2020,25(11):2000266

99 Conte C, Sogni F, Affanni P, et al. Vaccines against Coronaviruses: The State of the Art. Vaccines (Basel), 2020,8(2):E309

100 Ghaebi M, Osali A, Valizadeh H, et al. Vaccine development and therapeutic design for 2019-nCoV/ SARS-CoV-2: Challenges and chances. J Cell Physiol, 2020,235(12):9098-9109

$101 \mathrm{Wu}$ F, Wang A, Liu M, et al. Neutralizing antibody responses to SARS-CoV-2 in a COVID-19 recovered patient cohort and their implications. medRxiv, 2020: 20047365.

102 Sprangers MC, Lakhai W, Koudstaal W, et al. Quantifying adenovirus-neutralizing antibodies by luciferase transgene detection: addressing preexisting immunity to vaccine and gene therapy vectors. J Clin Microbiol, 2003,41(11):5046-5052

$103 \mathrm{Fu}$ X, Tao L, Zhang X. Comprehensive and systemic optimization for improving the yield of SARS-CoV-2 spike pseudotyped virus. Mol Ther Methods Clin Dev, 2021,20:350-356

104 Nie J, Li Q, Wu J, et al. Establishment and validation of a pseudovirus neutralization assay for SARS-CoV-2. Emerg Microbes Infect, 2020,9(1):680-686

105 Tang H, Kuhen KL, Wong-Staal F. Lentivirus replication and regulation. Annu Rev Genet. 1999,33:133-170
106 Whitelaw CBA. Transgenic livestock made easy. Trends Biotechnol, 2004,22(4):157-159

107 Lillico S, Vasey D, King T, et al. Lentiviral transgenesis in livestock. Transgenic Res, 2011,20(3):441-442

108 Picanco-Castro V, Sousa Russo-Carbolante EM de, Tadeu Covas D. Advances in lentiviral vectors: a patent review. Recent Pat DNA Gene Seq, 2012,6(2):82-90

109 Naldini L. Lentiviruses as gene transfer agents for delivery to non-dividing cells. Curr Opin Biotechnol, 1998,9(5):457-463

110 Kumar M, Keller B, Makalou N, et al. Systematic determination of the packaging limit of lentiviral vectors. Hum Gene Ther, 2001,12(15):1893-1905

111 Naldini L, Blömer U, Gage FH, et al. Efficient transfer, integration, and sustained long-term expression of the transgene in adult rat brains injected with a lentiviral vector. Proc Natl Acad Sci USA, 1996,93(21):1138211388

112 Reiser J, Harmison G, Kluepfel-Stahl S, et al. Transduction of nondividing cells using pseudotyped defective high-titer HIV type 1 particles. Proc Natl Acad Sci USA, 1996,93(26):15266-15271

113 Blömer U, Naldini L, Kafri T, et al. Highly efficient and sustained gene transfer in adult neurons with a lentivirus vector. J Virol, 1997,71(9):6641-6649

114 Kordower JH, Bloch J, Ma SY, et al. Lentiviral gene transfer to the nonhuman primate brain. Exp Neurol, 1999,160(1):1-16

115 Blesch A, Tuszynski MH. Transient growth factor delivery sustains regenerated axons after spinal cord injury. J Neurosci, 2007,27(39):10535-10545

116 Sinn PL, Sauter SL, McCray PB. Gene Therapy Progress and Prospects: Development of improved lentiviral and retroviral vectors -- design, biosafety, and production. Gene Ther, 2005,12(14):1089-1098

117 Sandrin V, Russell SJ, Cosset FL. Targeting retroviral and lentiviral vectors. Curr Top Microbiol Immunol, 2003,281:137-178

118 Cannon JR, Sew T, Montero L, et al. Pseudotypedependent lentiviral transduction of astrocytes or neurons in the rat substantia nigra. Exp Neurol, 2011, 228(1):41-52

119 Cronin J, Zhang XY, Reiser J. Altering the Tropism of Lentiviral Vectors through Pseudotyping. Curr Gene Ther, 2005,5(4):387-398

120 Lewis $\mathrm{P}$, Hensel M, Emerman M. Human immunodeficiency virus infection of cells arrested in the cell cycle. EMBO J, 1992,11(8):3053-3058

121 Wang J, Deng F, Ye G, et al. Comparison of lentiviruses pseudotyped with $\mathrm{S}$ proteins from coronaviruses and cell tropisms of porcine coronaviruses. Virol Sin, 2016, 31(1):49-56

122 Ren W, Qu X, Li W, et al. Difference in receptor usage between severe acute respiratory syndrome (SARS) coronavirus and SARS-like coronavirus of bat origin. J Virol, 2008,82(4):1899-1907

123 Kobinger GP, Limberis MP, Somanathan S, et al. Human immunodeficiency viral vector pseudotyped with the spike envelope of severe acute respiratory syndrome coronavirus transduces human airway epithelial cells and dendritic cells. Hum Gene Ther, 2007,18(5):413422 
124 Nie Y, Wang P, Shi X, et al. Highly infectious SARSCoV pseudotyped virus reveals the cell tropism and its correlation with receptor expression. Biochem Biophys Res Commun. 2004, 321(4):994-1000.

125 Escors D, Breckpot K. Lentiviral vectors in gene therapy: their current status and future potential. Arch Immunol Ther Exp (Warsz), 2010,58(2):107-119

126 Naldini L, Blömer U, Gallay P, et al. In vivo gene delivery and stable transduction of nondividing cells by a lentiviral vector. Science, 1996,272(5259):263-267

$127 \mathrm{Kim}$ VN, Mitrophanous K, Kingsman SM, et al. Minimal requirement for a lentivirus vector based on human immunodeficiency virus type 1. J Virol, 1998,72(1):811-816

128 Parolin C, Dorfman T, Palú G, et al. Analysis in human immunodeficiency virus type 1 vectors of cisacting sequences that affect gene transfer into human lymphocytes. J Virol, 1994,68(6):3888-3895

129 Fouchier RA, Simon JH, Jaffe AB, et al. Human immunodeficiency virus type 1 Vif does not influence expression or virion incorporation of gag-, pol-, and env-encoded proteins. J Virol, 1996,70(12):8263-8269

130 Zufferey R, Nagy D, Mandel RJ, et al. Multiply attenuated lentiviral vector achieves efficient gene delivery in vivo. Nat Biotechnol, 1997,15(9):871-875

131 Klaver B, Berkhout B. Comparison of 5' and 3' long terminal repeat promoter function in human immunodeficiency virus. J Virol, 1994,68(6):3830-3840

132 Yu SF, Rüden T von, Kantoff PW, et al. Self-inactivating retroviral vectors designed for transfer of whole genes into mammalian cells. Proc Natl Acad Sci USA, 1986,83(10):3194-3198

133 Laspia MF, Rice AP, Mathews MB. HIV-1 Tat protein increases transcriptional initiation and stabilizes elongation. Cell, 1989,59(2):283-292

134 Dull T, Zufferey R, Kelly M, et al. A third-generation lentivirus vector with a conditional packaging system. J Virol, 1998,72(11):8463-8471

135 Merten OW, Hebben M, Bovolenta C. Production of lentiviral vectors. Mol Ther Methods Clin Dev, 2016,3:16017

136 Fraga H. Firefly luminescence: a historical perspective and recent developments. Photochem Photobiol Sci, 2008,7(2):146-158

137 Uckert W, Pedersen L, Günzburg W. Green fluorescent protein retroviral vector: generation of high-titer producer cells and virus supernatant. Methods Mol Med, 2000,35:275-285

138 Heider S, Metzner C. Quantitative real-time single particle analysis of virions. Virology, 2014,462-463: 199-206

139 Geraerts M, Willems S, Baekelandt V, et al. Comparison of lentiviral vector titration methods. BMC Biotechnol, 2006,6:34

140 Case JB, Rothlauf PW, Chen RE, et al. Neutralizing Antibody and Soluble ACE2 Inhibition of a ReplicationCompetent VSV-SARS-CoV-2 and a Clinical Isolate of SARS-CoV-2. Cell Host Microbe, 2020,28(3):475-485. e5

141 Almahboub SA, Algaissi A, Alfaleh MA, et al. Evaluation of Neutralizing Antibodies Against Highly Pathogenic Coronaviruses: A Detailed Protocol for a Rapid Evaluation of Neutralizing Antibodies Using Vesicular Stomatitis Virus Pseudovirus-Based Assay. Front Microbiol, 2020,11:2020

142 Gan SD, Patel KR. Enzyme immunoassay and enzymelinked immunosorbent assay. J Invest Dermatol, 2013, 133(9):e12

143 Zhang N, Chen S, Wu J, et al. A lateral flow test detecting SARS-CoV-2 neutralizing antibodie. Available from: https://europepmc.org/article/PPR/PPR236206. 2020; [Accessed Aug 5, 2021].

144 Lequin RM. Enzyme immunoassay (EIA)/enzymelinked immunosorbent assay (ELISA). Clin Chem, 2005, 51(12):2415-2418

145 Banga Ndzouboukou JL, Zhang Y, Lei Q, et al. Human IgM and IgG Responses to an Inactivated SARS-CoV-2 Vaccine. Curr Med Sci, 2021, Nov 6:1-8. doi: 10.1007/ s11596-021-2461-8. Online ahead of print

146 Schöler L, Le-Trilling VTK, Eilbrecht M, et al. A Novel In-Cell ELISA Assay Allows Rapid and Automated Quantification of SARS-CoV-2 to Analyze Neutralizing Antibodies and Antiviral Compounds. Front Immunol, 2020,11:573526

147 Koczula KM, Gallotta A. Lateral flow assays. Essays Biochem, 2016,60(1):111-120

148 Mboowa G. Current and emerging diagnostic tests available for the novel COVID-19 global pandemic. AAS Open Res, 2020,3:8

149 Parolo C, Escosura-Muñiz A de la, Merkoçi A. Enhanced lateral flow immunoassay using gold nanoparticles loaded with enzymes. Biosens Bioelectron, 2013,40(1):412-416

150 Xu M, Wang D, Wang H, et al. COVID-19 diagnostic testing: Technology perspective. Clin Transl Med, 2020, 10(4):e158

151 Russo A, Minichini C, Starace M, et al. Current Status of Laboratory Diagnosis for COVID-19: A Narrative Review. Infect Drug Resist, 2020,13:2657-2665

152 Choudhary S, Sreenivasulu K, Mitra P, et al. Role of Genetic Variants and Gene Expression in the Susceptibility and Severity of COVID-19. Ann Lab Med, 2021,41(2):129-138

153 Lisboa Bastos M, Tavaziva G, Abidi SK, et al. Diagnostic accuracy of serological tests for covid-19: systematic review and meta-analysis. BMJ, 2020,370:m2516

154 Yun S, Ryu JH, Jang JH, et al. Comparison of SARSCoV-2 Antibody Responses and Seroconversion in COVID-19 Patients Using Twelve Commercial Immunoassays. Ann Lab Med, 2021,41(6):577-587

$155 \mathrm{Li} \mathrm{Z}$, Yi Y, Luo X, et al. Development and clinical application of a rapid IgM-IgG combined antibody test for SARS-CoV-2 infection diagnosis. J Med Virol, 2020,92(9):1518-1524

(Received Aug. 31, 2021; accepted Oct. 20, 2021) 